\title{
Persistent Hypertransaminasemia Uncovered Occult Limb-Girdle-Muscle Dystrophy-Type-2C in a Saudi Child
}

\author{
(1) Naglaa M. Kamal1,2, (1) Hamed A. Alghamdi2, (1) Abdulrahman Halabi2, (1) Abdullah O. Alharbi2, \\ (1) Muhammad Rafique2, (1) Kamel Abidi3, (1) Mortada H.H. El-Shabrawi1, (1) Ayman E. Eskander1, \\ (1) Hala Mansour1, (1) Laila M. Sherief4
}

${ }^{1}$ Cairo University Faculty of Medicine, Department of Pediatric Hepatology, Cairo, Egypt

${ }^{2}$ Alhada Armed Forces Hospital, Department of Pediatrics, Taif, Saudi Arabia

3 Tunis Al Manar University Faculty of Medicine, Department of Nephropediatric, Tunis, Tunisia

4 Zagazig University Faculty of Medicine, Department of Pediatrics, Zagazig, Egypt

\begin{abstract}
An asymptomatic 4.5 years-old Saudi girl was referred to the pediatric hepatology service with presumed liver disease because of the persistently elevated transaminases which were discovered accidently during routine laboratory workup. Alanine aminotransferase was $128 \mathrm{IU} / \mathrm{L}$ and aspartate aminotransferase was $143 \mathrm{IU} / \mathrm{L}$. Subsequent investigations in the hepatology clinic revealed normal hepatic workup. Muscle related work up revealed increased creatine phosphokinase, abnormal electromyography and motor nerve conduction. Muscle biopsy was suggestive of early stage muscular dystrophy, and analyses were compatible with limb-girdle-muscle-disease Type 2C. Further confirmation was reached by molecular genetic testing. This case demonstrates that increased transaminases do not always suggest liver disease, and occult muscle disease should always be taken into account while investigating patients with unexplained persistent hypertransaminasemia.

Keywords: Aminotransferases, children, creatine phosphokinase, myopathy
\end{abstract}

\section{Introduction}

It is well known that prolonged elevation of aminotransferases (ATs) is often suggestive of acute and chronic hepatic diseases (1). By their presence in various cells, especially hepatocytes, cardiac and skeletal myocytes, the rising of aspartate aminotransferase (AST) level is often attributed to a wide spectrum of clinical disorders. In contrast, the elevation of alanine aminotransferase (ALT) is known as a specific indicator of liver necrosis (2). However, in the absence of hepatic etiology more investigations are required to confirm muscular dystrophy, especially in patients with prolonged elevation of ATs. So, the coincidental finding of elevated ALT/AST may be the presenting sign of muscle disease in many children, and provides an opportunity for early diagnosis. Hence, early creatine phosphokinase (CPK) testing may provide the clue to the diagnosis of occult muscle disease in children with unexplained anicteric hypertransaminasemia (3).

\section{Case Report}

A 4.5 years old girl was examined by the general pediatrician because of upper respiratory tract infection. Routine blood analysis showed persistently elevated serum ALT concentrations. A hepatic problem was suspected and the patient was referred to the pediatric hepatology and gastroenterology clinic for further investigation of liver 
disease, which revealed normal serum bilirubin, gamma glutamyltranspeptidase, alkaline phosphate, albumin and prothrombin time. Other investigations for possible etiology of liver disease all came out negative including hepatitis markers, immunoglobulins, autoantibodies, alpha-1 antitrypsin (1.51 g/L-normal 0.98-1.96 g/L) and ceruloplasmin $(0.32$ $\mathrm{g} / \mathrm{L}$-normal 0.20-0.60 g/L). Liver doppler ultrasound was also normal. A muscular rather than a hepatic problem was suspected. For that reason liver biopsy was not done. More focus on the history, and examination of the muscular system uncovered that the child has baseline easy fatigability which progresses over the day. She had difficulty combing her hair and getting up from the ground. On examination bilateral calf muscles hypertrophy with positive Gower sign. Past medical history was uneventful with normal foetal movements and uncomplicated labour at 40 weeks of gestation. The girl's development was also uncomplicated; milestones were reached at the appropriate age, and family history was unremarkable. Muscle enzymes were studied and CPK levels were found to be highly elevated with serial CPK concentrations of 6651, $15831 \mathrm{U} / \mathrm{L}$. Electromyogram and Motor Nerve Conduction were done and reported to be suggestive of congenital myopathy. Her brother and sister were screened with CPK which came out normal. Muscle biopsy revealed some single atrophy as well as myopathic changes such as degeneration and regeneration of muscle fibers suggesting an early stage of muscular dystrophy. Immunohistochemical analyses were compatible with Sacroglycanopathy, possibly Limb Girdle Muscle Disease Type 2C (LGMD, 2C), which was further confirmed by molecular genetic testing. The patient was referred to the Pediatric Neurology Department. The time interval between the child's presentation to the Pediatric Hepatology and Gastroenterology Clinic and the diagnosis was nearly two months, although almost one year had passed from the initial coincidental finding of elevated AST and ALT until the final diagnosis.

\section{Discussion}

Persistently unexplained elevation of ATs is most commonly observed at the present time. A clear elevation of more than ten times the normal range is a well-validated index of cytolysis liver, but a more moderate rise can also reveal a metabolic muscle disease whose diagnosis is sometimes difficult to establish $(1,2)$. ATs are two cytosolic enzymes: ALT essentially has hepatic origin, and secondarily, muscle and kidney (3) origin. AST has a much wider distribution within the liver, but also in the heart, skeletal muscle, kidney and brain. Their physiological levels in plasma are between 5 and 50 IU/L but they depend on the technical laboratory, population reference and the selected distribution range (4). In line with other reports, the increased transaminases in our case also resulted in referral to the Hepatology and Gastroenterology Department for examination and evaluation; and muscle disease did not cross the mind of the assessing physician
$(5,6)$. The findings in our patient demonstrate that elevated serum AST and ALT in the absence of signs and symptoms of liver disease should lead to the consideration of occult muscle disease as a probable source. This assumption should be considered only after having eliminated any liver disease and carrying out a thorough interview and a complete physical examination with some laboratory tests. That is why in our case a more focused history and examination of muscular system was carried out, revealing that the child had easy fatigability, which progressed over the day with difficulty combing her hair and getting up from the ground. Calf muscles were hypertrophied with positive Gower sign and high CPK suggesting muscle dystrophy. This was further confirmed by muscle biopsy and molecular genetic testing. Actually, elevated serum ATs may not only indicate hepatic disease but could also stem from muscle or other cellular damage (7). Both CPK level and the ratio between AST and ALT may be of help in differentiating between muscle and liver cell damage (8). The findings in our patient indicate that elevated AST and ALT in the absence of signs of liver disease should lead to the consideration of occult muscle disease. So, the measurement of serum CPK and a careful physical examination are the most useful and cost effective means to correctly identify these patients before carrying out invasive tests such as liver biopsy. This phenomenon has been described in many case reports $(4,8)$ and in a series of five male Taiwanese patients with raised serum ALT and AST in whom signs and symptoms of hepatic disease were absent but evidence of neuromuscular dysfunction was detectable on clinical examination. The ages of these children were between 4 months and 5.5 years. None of the neurological findings were remarkable. The initial ALT and AST values were 114-581 U/L and 183-700 IU/L, respectively. Serum CPK was checked first after 0 to 30 months followup and found to be markedly elevated (range, $10.557 \mathrm{IU} / \mathrm{L}$ to $62.508 \mathrm{IU} / \mathrm{L}$ ). Muscle biopsies in all of the five cases showed degenerating and regenerating myofibers with interstitial fibrosis, and genetic studies showed deletions in the $D M D$ gene (9). The question thus remains, "What is wise in cases where no or only mild symptoms of the myopathic disease are present?" Thinking of alternative sources of alanine and aspartate ATs can help in avoiding such clinical pitfalls and spare families the anxiety and trauma of unnecessary investigations and delays in diagnosis, which may have prognostic implications. We recommend that when there are no clear clinical findings of hepatic or muscle disease in a child with persistently elevated AST and ALT, a CPK estimation on serum would be a wise step before going to invasive and expensive hepatic investigations including liver biopsy. This case report emphasizes that increased ATs do not always suggest liver disease. Occult muscle diseases should be taken into account in patients with unexplained long-lasting hypertransaminasemia, and therefore the measurement of serum CPK activity and muscle biopsy should be done early for a more correct diagnosis to avoid invasive and expensive investigations such as liver biopsy. 


\section{Ethics}

Informed Consent: Written informed consent was obtained from the parents for contribution of their child in the current case report and for publication.

Peer-review: Externally peer-reviewed.

\section{Authorship Contributions}

Surgical and Medical Practices: N.M.K., H.A.A., A.O.A., A.H., Concept: N.M.K., Design: N.M.K., Data Collection or Processing: M.R., K.A., Analysis or Interpretation: M.R., K.A., Literature Search: N.M.K., M.H.H.E.S., A.E.E., H.M., L.M.S., Writing: N.M.K., M.H.H.E.S., A.E.E., H.M., L.M.S.,

Conflict of Interest: No conflict of interest was declared by the authors.

Financial Disclosure: The authors declared that this study received no financial support.

\section{References}

1. Giannini EG, Testa R, Savarino V. Liver enzyme alteration: a guide for clinicians. CMAJ 2005;172:367-79.

2. Nathwani RA, Pais S, Reynolds TB, Kaplowitz N. Serum alanine aminotransferase in skeletal muscle diseases. Hepatology 2005;41:380-2.
3. Papadimas GK, Areovimata A, Papadopoulos C, Manta P. Re: Consider muscle disease in children with elevated transaminase. J Am Board Fam Med 2012;25:937

4. Dibek Misirlioğlu E, Albayrak M, Aliefendioğlu D. Elevations of serum aminotransferase in muscular dystrophy. Turk J Gastroenterol 2008;19:202.

5. Kohli R, Harris DC, Whitington PF. Relative elevations of serum alanine and aspartate aminotransferase in muscular dystrophy. J Pediatr Gastroenterol Nutr 2005;41:121-4.

6. Lash T, Kraemer RR. Elevated liver enzymes indicating a diagnosis of limb-girdle muscular dystrophy. J Gen Intern Med 2014;29:813-5.

7. McMillan HJ, Gregas M, Darras BT, Kang PB. Serum transaminase levels in boys with Duchenne and Bucker muscular dystrophy. Pediatrics 2011;127:132-6.

8. Wright MA, Yang ML, Parsons JA, Westfall JM, Yee AS. Consider muscle disease in children with elevated transaminase. J Am Board Fam Med 2012;25:536-40.

9. Lin $Y C$, Lee WT, Huang SF, Young $C$, Wang PJ, Shen YZ. Persistent hypertransaminasemia as the presenting finding of muscular dystrophy in childhood. Acta Paediatr Taiwan 1999;40:424-9. 